\title{
Computational Homogenization for Laminated Ferromagnetic Cores in Magnetodynamics
}

\author{
I. Niyonzima ${ }^{1}$, R. V. Sabariego ${ }^{1}$, P. Dular ${ }^{1,2}$, F. Henrotte ${ }^{3}$ and C. Geuzaine ${ }^{1}$ \\ ${ }^{1}$ Dept. of Electrical Engineering and Computer Science, University of Liège, Belgium \\ ${ }^{2}$ Fonds de la Recherche Scientifique - FNRS, Belgium \\ ${ }^{3}$ Institute of Mechanics, Materials and Civil Engineering, Université Catholique de Louvain
}

\begin{abstract}
In this paper, we investigate the modeling of ferromagnetic multiscale materials. We propose a computational homogenization technique based on the heterogeneous multiscale method (HMM) that includes both eddy-current and hysteretic losses at the mesoscale. The HMM comprises: 1) a macroscale problem that captures the slow variations of the overall solution; 2) many mesoscale problems that allow to determine the constitutive law at the macroscale. As application example, a laminated iron core is considered.
\end{abstract}

Index Terms - Computational homogenization, multiscale methods, ferromagnetic cores, magnetodynamics, hysteresis, finite-element methods.

\section{INTRODUCTION}

$\mathbf{T}$ HE ferromagnetic cores of electromagnetic devices are often laminated for reducing the losses. In some applications, a precise finite-element analysis of such stacked structures is crucial and homogenization techniques indispensable. Further the nonlinear and irreversible behaviour exhibited by ferromagnetic materials must be accounted for.

The Heterogeneous Multiscale Method (HMM) [6] is a popular methodology, mainly in mechanical and thermal problems, for studying multiscale materials with a reduced computational cost. This method has lately gained interest in electromagnetism. In particular, a finite element nonlinear multiscale homogenization method for magnetostatic problems was proposed in [11]. In this paper, the computational multiscale method is extended in order to include eddy currents and magnetic hysteresis. The approach is inspired by the multiscale technique developed in [12] for an elasto-plastic material governed by an irreversible constitutive law.

\section{Magnetodynamic PROBlem}

The magnetodynamic problem in a bounded domain $\Omega=$ $\Omega_{c} \cup \Omega_{c}^{C} \in \mathbb{R}^{3}$ is defined by the following Maxwell equations and constitutive laws [4]:

$$
\begin{array}{cc}
\operatorname{curl} \underline{h}=\underline{j}, & \operatorname{curl} \underline{e}=-\partial_{t} \underline{b}, \\
\underline{h}=\underline{h}(\underline{b}), & \underline{j}=\underline{\sigma} \underline{e}+\underline{j}_{s},
\end{array}
$$

with $\underline{h}$ the magnetic field, $\underline{b}$ the magnetic flux density, $\underline{j}$ the electric current density, $\underline{j}_{s}$ the imposed electric current density (source) and $\underline{e}$ the electric field. The electric linear law involves $\underline{\sigma}$, the (anisotropic) electric conductivity. The magnetic law can be linear, nonlinear reversible or nonlinear irreversible (i.e. with hysteresis). The domain $\Omega_{c}$ contains conductors whereas the domain $\Omega_{c}^{C}$ contains insulators and the inductors $\Omega_{s}$ where the sources are imposed. Proper boundary conditions must also be imposed.

In this paper, we use the $\underline{a}-v$ formulation and write $\underline{b}$ and $\underline{e}$ as:

$$
\underline{b}=\operatorname{curl} \underline{a}, \quad \underline{e}=-\partial_{t} \underline{a}-\operatorname{grad} v,
$$

Corresponding author: I. Niyonzima (e-mail: iniyonzima@doct.ulg.ac.be) with $\underline{a}$ the magnetic vector potential and $v$ the electric scalar potential defined only in $\Omega_{c}$. The weak form of ( 1 a) reads [4]: find $\underline{a}$ and $v$ such that:

$$
\begin{gathered}
\left(\underline{h}(\operatorname{curl} \underline{a}), \operatorname{curl} \underline{a}^{\prime}\right)_{\Omega}+\left(\underline{\underline{\sigma}} \partial_{t} \underline{a}, \underline{a}^{\prime}\right)_{\Omega_{c}}+\left(\underline{\left.\underline{\sigma} \operatorname{grad} v, \underline{a}^{\prime}\right)_{\Omega_{c}}}\right. \\
=-\left\langle\boldsymbol{n} \wedge \underline{h}, \underline{a}^{\prime}\right\rangle_{\Gamma_{h}}+\left(\underline{j} \underline{s}, \underline{a}^{\prime}\right)_{\Omega_{s}} \\
\left(\underline{\underline{\sigma}} \partial_{t} \underline{a}, \operatorname{grad} v^{\prime}\right)_{\Omega_{c}}+\left(\underline{\underline{\sigma}} \operatorname{grad} v, \operatorname{grad} v^{\prime}\right)_{\Omega_{c}}=\left\langle\boldsymbol{n} \cdot \underline{j}, v^{\prime}\right\rangle_{\Gamma_{g}}
\end{gathered}
$$

holds for all test functions $\underline{a}^{\prime}$ and $v^{\prime}$ in an appropriate function space. Surfaces $\Gamma_{h}$ and $\Gamma_{g}$ are the regions where a natural boundary condition on $\underline{h}$ is imposed and the cross section of $\Omega_{c}$ which is crossed by the current, respectively. In the following, for the sake of conciseness, we omit these boundary terms.

\section{Multiscale MODEL}

Fine-scale heterogeneities in a multiscale material induce rapid spatial variations of the computed fields. We define the exponent $\varepsilon=\frac{l}{L}$ which is the ratio between the smallest scale $l$ and the scale of the material or the characteristic length of external loadings $L$ and use it to denote quantities with rapid spatial variations.

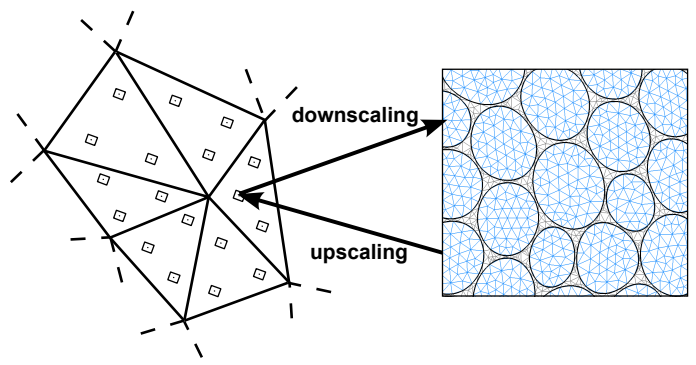

Fig. 1. Scale transitions between macroscale (left) and mesoscale (right) problems. Downscaling (Macro to meso): obtaining proper boundary conditions and the source terms for the mesoscale problem from the macroscale solution. Upscaling (meso to Macro): effective quantities for the macroscale problem calculated from the mesoscale solution [11].

The multiscale problem can be solved in the whole domain using e.g. the finite element method. However this is very 
expensive in terms of memory and computation time due to the need of discretizing the unknown field at the smallest scale $\varepsilon$. A finite element computational homogenization method allows to overcome this problem and offers a good compromise between accuracy and computational cost. The method, based on the scale separation assumption $(\varepsilon \ll 1)$, is illustrated in Fig. 1.

A macroscale problem is defined on a coarse mesh covering the entire domain and many mesoscale problems are defined on small, finely meshed areas around some points of interest of the macroscale mesh (e.g. numerical quadrature points). Hereafter, the variables $\boldsymbol{x}=\left(x_{1}, x_{2}, x_{3}\right)$ and $\boldsymbol{y}=\left(y_{1}, y_{2}, y_{3}\right)$ are the macroscale and the mesoscale spatial positions, respectively and $\boldsymbol{y}=\boldsymbol{x}-\boldsymbol{x}_{b}$ is only defined on the cell domains with the origin located at the barycenter $\boldsymbol{x}_{b}$ of the cell. We define operators with respect to these variables. For instance, $\operatorname{curl}_{x}$ and $\operatorname{curl}_{y}$ denote curl operators with respect to $\boldsymbol{x}$ and $\boldsymbol{y}$. The subscripts $M, m$ and $c$ refer to the macroscale, the total and the correction mesoscale quantities, respectively. Only mesoscale quantities depend on the $\boldsymbol{y}$ coordinates. The average of $f^{\varepsilon}$ over $\Omega_{m}$ (with $\left|\Omega_{m}\right|$ the volume of the cell) is denoted by $<\underline{f}^{\varepsilon}>_{\Omega_{m}}=\frac{1}{\left|\Omega_{m}\right|} \int_{\Omega_{m}} f^{\varepsilon} \mathrm{d} y$.

The two-scale asymptotic expansion method [3] is used for homogenizing the linear electric law. Indeed, this theory is suitable for linear and weakly nonlinear materials. Further, we resort the two-scale convergence theory, better suited for the homogenization of highly nonlinear problems in order to obtain the homogenized model for the Maxwell equations in magnetodynamics. For more information about the general two-scale convergence theory, see [1] and references therein and [13], [14] for electromagnetism.

Applying this theory, it can be shown that the fields $\underline{h}^{\varepsilon}, \underline{b}^{\varepsilon}$, $\underline{e}^{\varepsilon}$ and $j^{\varepsilon}$ converge (in the two-scale sense) to some macroscale fields $\underline{h}_{M}, \underline{b}_{M}, \underline{e}_{M}$ and $\underline{j}_{M}$, respectively. Moreover, the finescale problem can be replaced by 1) a macroscale problem; 2) many mesoscale problems. The transfer of information between these problems and the weak formulations at both scales are detailed hereafter.

\section{A. Downscaling}

Mesoscale field quantities (subscript $m$ ) are governed by (1 a-b)-(2a-b) and $j_{s} \equiv 0$. The two-scale convergence theory allows us to express the curl of the electric field at the mesoscale in terms of the electric field at the macroscale and a mesoscale correction, such that $\operatorname{curl}_{y} \underline{e}_{m}^{\varepsilon}=\operatorname{curl}_{x} \underline{e}_{M}+\operatorname{curl}_{y} \underline{e}_{c}^{\varepsilon}$. Using the Faraday law at the macroscale together with the vector identity $\operatorname{curl}_{y}\left(\partial_{t} b_{M} \wedge \boldsymbol{y}\right)=(n-1) \partial_{t} b_{M}(n=2,3$ for $2 \mathrm{D}$ and $3 \mathrm{D}$ problems, respectively) we can write

$$
\operatorname{curl}_{y} \underline{e}_{m}^{\varepsilon}=\operatorname{curl}_{y}\left(\underline{e}_{c}^{\varepsilon}+\underline{e}_{M}-\kappa\left(\partial_{t} \underline{b}_{M} \wedge \boldsymbol{y}\right)\right)
$$

with $\kappa=(n-1)^{-1}$, since $\operatorname{curl}_{y} \underline{e}_{M} \equiv 0$. This provides a natural development of $\underline{e}_{m}^{\varepsilon}$ in terms of a local, rapidly fluctuating component and a large scale component, for which we impose that $<\underline{e}_{m}^{\varepsilon}>_{\Omega_{m}}=\underline{e}_{M}$. Similar developments have been proposed in [7] and [9] for the electric and the magnetic fields in linear cases. Unlike these papers, we keep the multiscale form (i.e. we do not consider the two-scale homogenized form) for the Ampere equation $\operatorname{curl}_{y} \underline{h}_{m}^{\varepsilon}=\underline{j}_{m}^{\varepsilon}$.
We can thus use nonlinear and hysteretic magnetic laws $\underline{h}-\underline{b}$ for the mesoscale model. We develop $\underline{e}_{m}^{\varepsilon}$ and $\underline{b}_{m}^{\varepsilon}$ as follows:

$$
\left\{\begin{array}{l}
\underline{e}_{m}^{\varepsilon}=-\partial_{t} \underline{a}_{c}^{\varepsilon}-\operatorname{grad}_{y} v_{c}^{\varepsilon}+\underline{e}_{M}-\kappa\left(\partial_{t} \underline{b}_{M} \wedge \boldsymbol{y}\right) \\
\underline{b}_{m}^{\varepsilon}=\operatorname{curl}_{y} \underline{a}_{c}^{\varepsilon}+\underline{b}_{M}
\end{array}\right.
$$

where the macroscale fields $\underline{b}_{M}$ and $\underline{e}_{M}$ are the source terms for the mesoscale problem. Boundary conditions for the mesoscale problem are also determined so as to respect the two-scale convergence of the physical fields: the convergence of the magnetic flux density $\underline{b}$ leads to a periodicity condition for the tangential component of the correction term of the magnetic vector potential $\underline{a}_{c}^{\varepsilon}$, i.e.

$$
<\underline{b}_{m}^{\varepsilon}>_{\Omega_{m}}=\underline{b}_{M} \Longrightarrow \oint_{\Gamma_{m}} \boldsymbol{n} \wedge \underline{a}_{c}^{\varepsilon} \mathrm{d} y=0
$$

A condition of periodocity must also be imposed for the term $v_{c}^{\varepsilon}$. The convergence of the electric field $\underline{e}$ leads to

$$
<\underline{e}_{m}^{\varepsilon}>_{\Omega_{m}}=\underline{e}_{M} \Longrightarrow \int_{\Omega_{m}} \underline{e}_{c}^{\varepsilon} \mathrm{d} y=0,
$$

for the correction term of the electric field $\underline{e}_{c}^{\varepsilon}$.

\section{B. Upscaling}

The upscaling consists in computing the missing constitutive laws $\underline{\underline{\sigma}}_{M}, \underline{h}_{M}\left(\underline{b}_{M}\right)$ together with $\frac{\partial \underline{h}_{M}}{\partial \underline{b}_{M}}$ at the macroscale using the mesoscale fields.

Due to the linearity of the electric law, the asymptotic expansion theory [3] can be applied. Therefore, we compute once for all the homogenized electric conductivity by solving a cell problem. A similar approach was also adopted in [5]. The electric conductivity is then upscaled by means of:

$$
\left(\underline{\underline{\sigma}}_{M}\right)_{i j}=\left\langle\left(\underline{\underline{\sigma}}_{m}^{\varepsilon}\right)_{i j}-\left(\underline{\underline{\sigma}}_{m}^{\varepsilon}\right)_{i k} \frac{\partial \chi_{j}^{\varepsilon}}{\partial y_{k}}\right\rangle_{\Omega_{m}}
$$

where the periodic functions $\chi_{j}^{\varepsilon}$ are solutions of the cell problem: find $\chi_{j}^{\varepsilon}$ such that:

$$
\int_{\Omega_{m}}\left(\operatorname{grad}_{y} \psi^{\varepsilon^{\prime}}\right)^{T} \underline{\sigma}_{m}^{\varepsilon}\left(\operatorname{grad}_{y} \chi_{j}^{\varepsilon}-\boldsymbol{e}_{j}\right) \mathrm{d} y=0
$$

holds for all $\psi^{\varepsilon^{\prime}}$ in an appropriate function space. The vector $\boldsymbol{e}_{j}$ is the unit vector in the $j^{t h}$ spatial direction.

The upscaling of the nonlinear magnetic law is performed by simple average as a consequence of the two-scale convergence of $\underline{h}^{\varepsilon}$ :

$$
<\underline{h}_{m}^{\varepsilon}>_{\Omega_{m}}=\underline{h}_{M} .
$$

The tangent matrix $\frac{\partial \underline{h}_{M}}{\partial \underline{b}_{M}}$ for the Newton-Raphson scheme is obtained by finite differences [10].

\section{Weak formulations}

The macroscale formulation reads: find $\underline{a}_{M}$ and $v_{M}$ such that

$$
\begin{gathered}
\left(\underline{h}_{M}, \operatorname{curl} \underline{a}_{M}^{\prime}\right)_{\Omega}+\left(\underline{\underline{\sigma}}_{M} \partial_{t} \underline{a}_{M}, \underline{a}_{M}^{\prime}\right)_{\Omega_{c}}+ \\
\left(\underline{\underline{\sigma}}_{M} \operatorname{grad}_{x} v_{M}, \underline{a}_{M}^{\prime}\right)_{\Omega_{c}}=\left(\underline{j}_{s}, \underline{a}_{M}^{\prime}\right)_{\Omega_{s}} \\
\left(\underline{\underline{\sigma}}_{M} \partial_{t} \underline{a}_{M}, \operatorname{grad}_{x} v_{M}^{\prime}\right)_{\Omega_{c}}=-\left(\underline{\underline{\sigma}}_{M} \operatorname{grad}_{x} v_{M}, \operatorname{grad}_{x} v_{M}^{\prime}\right)_{\Omega_{c}}
\end{gathered}
$$




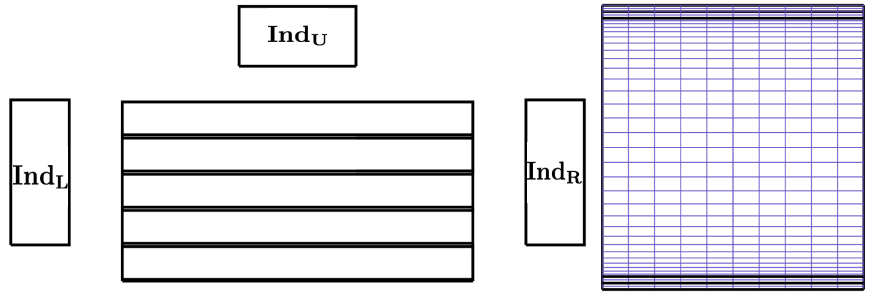

Fig. 2. Left: geometry used for the validation of the model, taking advantage of symmetry. For the sake of visibility, only 5 laminations (out of 15) are represented. $\operatorname{Ind}_{\mathrm{L}}, \mathrm{Ind}_{\mathrm{R}}$ and $I n \mathrm{I}_{\mathrm{U}}$ are the inductors located on the left, the right and the top sides of the stack respectively. Right: geometry and mesh used for mesoscale computations.

hold for all test functions $\underline{a}_{M}^{\prime}$ and $v_{M}^{\prime}$ in appropriate function spaces.

The mesoscale formulation reads:

find $\underline{a}_{c}^{\varepsilon}$ and $v_{c}^{\varepsilon}$ such that

$$
\begin{gathered}
\left(\underline{h}_{m}^{\varepsilon}\left(\operatorname{curl}_{y} \underline{a}_{c}^{\varepsilon}+\underline{b}_{M}\right), \operatorname{curl}_{y} \underline{a}_{c}^{\varepsilon^{\prime}}\right)_{\Omega_{m}}+\left(\underline{\underline{\sigma}}_{m}^{\varepsilon} \partial_{t} \underline{a}_{c}^{\varepsilon}, \underline{a}_{c}^{\varepsilon^{\prime}}\right)_{\Omega_{m_{c}}}+ \\
\left(\underline{\underline{\sigma}}_{m}^{\varepsilon} \operatorname{grad}_{y} v_{c}^{\varepsilon}, \underline{a}_{c}^{\varepsilon^{\prime}}\right)_{\Omega_{m_{c}}}=\left(\underline{\underline{\sigma}}_{m}^{\varepsilon}\left(\underline{e}_{M}-\kappa \partial_{t} \underline{b}_{M} \wedge \boldsymbol{y}\right), \underline{a}_{c}^{\varepsilon^{\prime}}\right)_{\Omega_{m_{c}}} \\
\left(\underline{\underline{\sigma}}_{m}^{\varepsilon} \partial_{t} \underline{a}_{c}^{\varepsilon}, \operatorname{grad}_{y} v_{m}^{\prime} \varepsilon\right)_{\Omega_{m c}}+\left(\underline{\underline{\sigma}}_{m}^{\varepsilon} \operatorname{grad}_{y} v_{c}^{\varepsilon}, \operatorname{grad}_{y} v_{m}^{\prime}\right)_{\Omega_{m c}}= \\
\left(\underline{\underline{\sigma}}_{m}^{\varepsilon}\left(\underline{e}_{M}-\kappa \partial_{t} \underline{b}_{M} \wedge \boldsymbol{y}\right), \operatorname{grad}_{y} v_{m}^{\prime}\right)_{\Omega_{m c}}+\left\langle\boldsymbol{n} \cdot \underline{j}_{M}, v_{m}^{\prime}\right\rangle_{\Gamma_{g m}}
\end{gathered}
$$

hold for all test functions $\underline{a}_{m}^{\prime}$ and $v_{m}^{\prime}$ in appropriate function spaces. Domains $\Omega_{m c}$ and $\Gamma_{g m}$ are the conducting part of the mesoscale domain and the boundary of $\Omega_{m c}$, respectively. The electric current density $\underline{j}_{M}=\underline{\sigma}_{M} \underline{e}_{M}$ is obtained from the macroscale solution.

\section{Application ExAmple}

We consider a two-dimensional model of a laminated core $(16.45 \mathrm{~mm} \times 16.45 \mathrm{~mm})$ consisting of 30 laminations (thickness $d_{l}=0.5 \mathrm{~mm}$, conductivity $\sigma=5 \mathrm{MS} / \mathrm{m}$ ) and 29 insulation layers (thickness $d_{0}=0.05 \mathrm{~mm}$ ), so that $\varepsilon \approx 0.033$. The filling factor is $\lambda=d_{l} /\left(d_{l}+d_{0}\right)=0.91$. Taking advantage of the symmetry, only half of the model has been studied (See Fig. 2). Note that as we consider perferctly isolated laminations, there are no currents flowing from one lamination to the other. Indeed, $\left(\underline{\sigma}_{M}\right)_{x_{2} x_{2}}=0$, where $x_{2}$ is the direction normal to the laminations so that no eddy currents are to be accounted for at the macroscale $\left(\underline{j}_{M}=\underline{0}\right.$ and $\left.\underline{e}_{M}=\underline{0}\right)$. The insulation material is linear (with $\mu_{r}=1$ ). The lamination material is governed by the following magnetic laws:

- nonlinear: $\underline{h}^{\varepsilon}\left(\underline{b}^{\varepsilon}\right)=\left(\alpha+\beta \exp \left(\gamma\left\|\underline{b}^{\varepsilon}\right\|^{2}\right)\right) \underline{b}^{\varepsilon}$ with $\alpha=$ $388, \beta=0.3774$ and $\gamma=2.97$.

- Jiles-Atherton hysteresis model with parameters $m_{s}=$ $1,145,500 \mathrm{~A} / \mathrm{m}, a=59 \mathrm{~A} / \mathrm{m}, k=99 \mathrm{~A} / \mathrm{m}, c=0.55$ and $\alpha=1.3 \times 10^{-4}$ [2].

A sinusoidal electric current density with amplitudes $\underline{j}_{s L}=$ $\underline{j}_{s R}$ and $\underline{j}_{s U}$ is imposed in inductors $\operatorname{Ind}_{\mathrm{L}}$, $\operatorname{Ind}_{\mathrm{R}}$ and $\operatorname{Ind}_{\mathrm{U}}$, respectively: 1) nonlinear case with frequency $f=500 \mathrm{~Hz}$ and amplitudes $\underline{j}_{s L}=2.5 \times 10^{8} \mathrm{~A} / \mathrm{m}^{2}$ and $\underline{j}_{U}=2.5 \times 10^{7} \mathrm{~A} / \mathrm{m}^{2}$; 2) hysteretic case with $f=20 \mathrm{~Hz}, \underline{j}_{s L}=10^{8} \mathrm{~A} / \mathrm{m}^{2}$ and $\underline{j}_{U}=$ $10^{7} \mathrm{~A} / \mathrm{m}^{2}$.
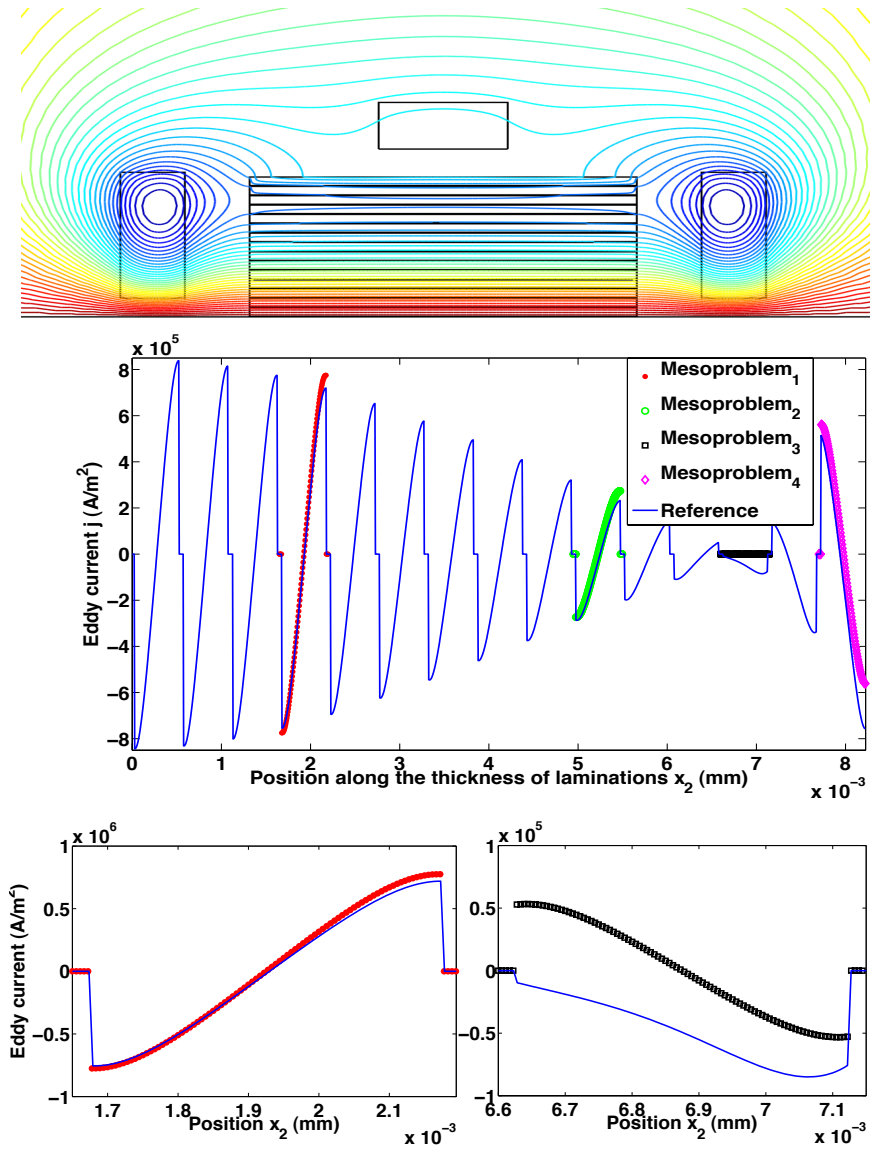

Fig. 3. Nonlinear case - Top: Magnetic vector potential $\underline{a}$ ( $x_{3}$ component). Middle: Eddy currents $\underline{j}_{m}^{\varepsilon}\left(x_{1}\right.$ component $)$ for a cut at $x_{1}=0.275 \mathrm{~mm}$ $(t=0.0004 \mathrm{~s}$ ). Comparison between the FE reference model (continuous line) and 4 mesoscale solutions defined with $x_{2}$ in intervals $[1.65,2.195] \mathrm{mm}$, $[4.95,5.5] \mathrm{mm},[6.6,7.15] \mathrm{mm},[7.7,8.225] \mathrm{mm}$, respectively. Bottom: Zoom of the eddy currents around the mesoscale problems 1 and 3 .

The reference solution is obtained by a brute force approach, i.e. solving a finite element (FE) problem on an extremely fine mesh of the whole stack consisting of 30 layers of 81 quadrangles for each lamination and 4 layers of 81 quadrangles for each insulation layer (i.e. 41,148 elements for the conductors and the insulation layers). The mesoscale problems are solved on square domains comprising one lamination and one insulation layer (Fig. 2). Each lamination is discretized with 30 layers of 10 quadrangles and each insulation layer with 8 layers of 10 quadrangles. The coarse mesh of the lamination stack contains 225 and 300 quadrangular elements, respectively for the nonlinear and the hysteresis problems with one integration point per element. The computational problem is solved over one period with 20 time steps per period for the nonlinear problem and two periods with 120 time steps per period for the hysteresis problem.

\section{A. Nonlinear case}

Results obtained using the computational homogenization approach are compared to those obtained using a brute force approach. Flux lines obtained with the FE reference model are depicted in Fig. 3. Top. These lines show the presence of an area in the laminations where the fields weaken before 

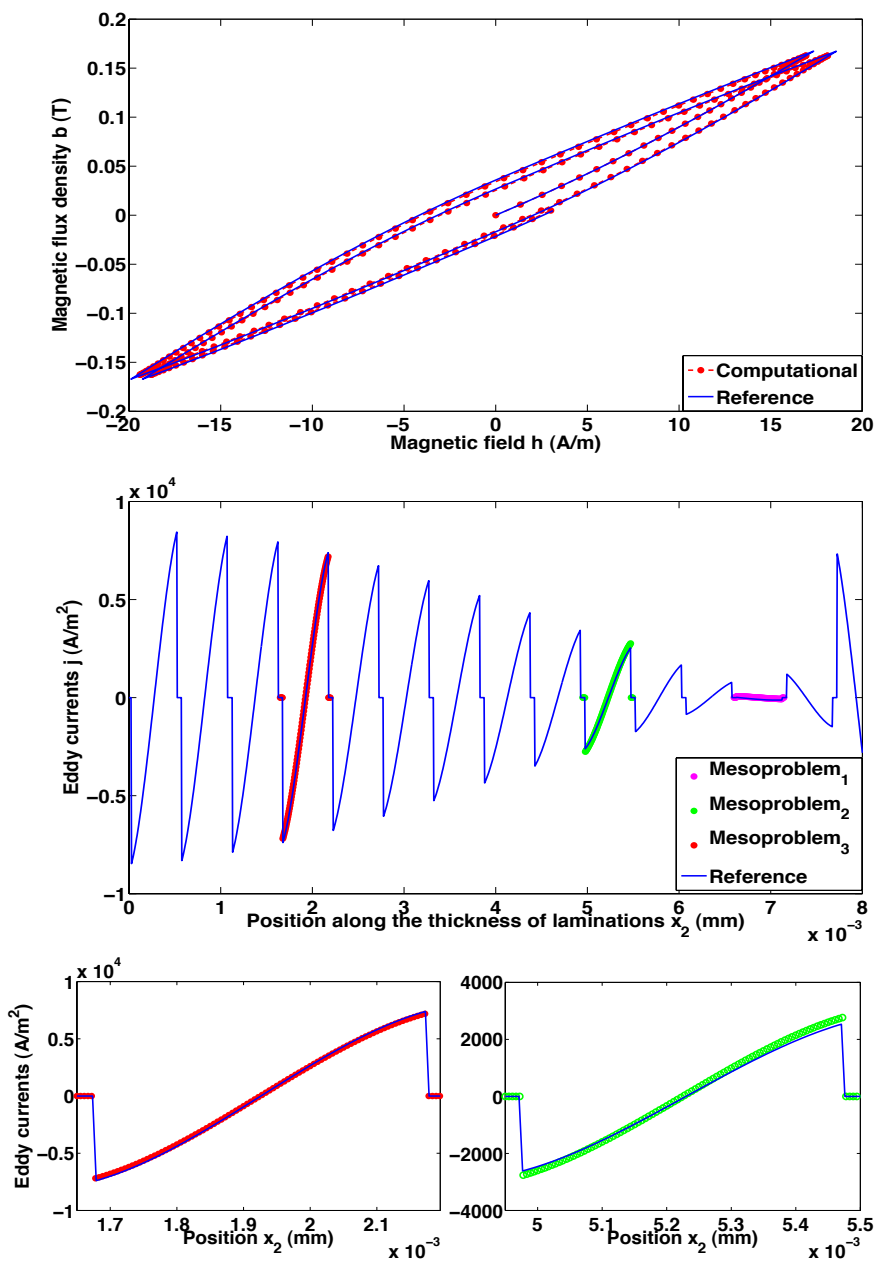

Fig. 4. Hysteresis case - Top: Reference and computational $h b$-hysteretic curves for a point located at $x_{1}=1.65 \mathrm{~mm}, x_{2}=-3.7 \mathrm{~mm}$. Middle: Eddy currents $j_{m}^{\varepsilon}$ ( $x_{3}$ component) for a cut at $x_{1}=3.7 \mathrm{~mm}(t=0.0104 \mathrm{~s})$ for FE reference model (continuous line) and 4 mesoscale problems defined with $x_{2}$ in intervals $[1.65,2.195] \mathrm{mm},[4.95,5.5] \mathrm{mm},[6.6,7.15] \mathrm{mm}$, $[7.7,8.225] \mathrm{mm}$, respectively. Bottom: Zoom of the eddy currents around the mesoscale problems 1 and 3 .

changing direction. Values of the local fields obtained on a cut at $x_{1}=0.275 \mathrm{~mm}$ show a good agreement between the reference and the local mesoscale solutions (Fig. 3. Middle and Bottom). Small discrepancies are noticeable in regions with small eddy currents. There are also discrepancies in the extreme layers as they do not have the same environment as the rest of laminations. We have observed the same behavior for $\underline{b}$ : the agreement between the macroscale (homogenized) field is in good agreement with the reference solution.

\section{B. Hysteresis case}

The reference and the computational $\underline{h b}$ hysteretic curves at point $x_{1}=1.65 \mathrm{~mm}$ (Fig. 4 . Top), as well as the values of the local fields obtained on a cut at $x_{1}=3.7 \mathrm{~mm}$ (Fig. 4. Middle and Bottom) are in excellent agreement.

\section{Conclusions and Perspectives}

In this paper, we have developed an HMM-based computational homogenization technique for dealing with magnetodynamic problems. The model accounts for the eddy current and hysteresis losses at the mesoscale and provides macroscale fields that approximate well the actual solution of the problems. Furthermore, local data can also be recovered by means of solutions of mesoscale problems at points of interest. Further developments will concern the development of $\underline{h}$-conform formulations, the inclusion of an energy-consistent hysteresis model [8] in the mesoscale model, and the reduction of the computational cost through parallelization of the computation of mesoscale problems.

\section{ACKNOWLEDGMENT}

This work has been supported by the Belgian French Community (ARC 09/14-02) and the Belgian Science Policy (IAP P7/02). I. Niyonzima is a fellowship beneficiary with the Belgian Research Training Fund for Industry and Agriculture (FRIA).

\section{REFERENCES}

[1] G. Allaire, Homogenization and the two-scale convergence, SIAM Journal on Mathematical Analysis, vol. 23, pp. 1482-1518, 1992.

[2] A. Benabou, S. Clnet and F. Piriou, Comparison of Preisach and Jiles-Atherton models to take into account hysteresis phenomenon for finite element analysis, Journal of Magnetism and Magnetic Materials, vol. 261, pp. 305-310, 2003.

[3] A. Bensoussan, J. L. Lions, G. Papanicolau Asymptotic Analysis for Periodic Structures, Studies in Mathematics and Its Applications., NorthHolland, 1978.

[4] A. Bossavit, Computational Electromagnetism. Variational Formulations, Complementarity, Edge Elements, Academic Press, 1998.

[5] O. Bottauscio, V. C. Piat, M. Chiampi, M. Codegone and A. Manzin, Nonlinear Homogenization Technique for Saturable Soft Magnetic Composites, IEEE Transactions on Magnetics, vol. 44, no. 11, pp. 2955-2958, 2008.

[6] W. E, B. Engquist, X. Li, W. Ren and E. Vanden-Eijnden, Heterogeneous Multiscale Methods: A Review, Communications in Computational Physics, vol. 2, n. 3, pp. 367-450, 2007.

[7] M. El Feddi, Z. Ren, A. Razek and A.Bossavit, Homogenization Technique for Maxwell Equations in Periodic Structure, IEEE Transactions on Magnetics, vol. 33, no. 2, pp. 1382-1385, 1997.

[8] V. Francois-Lavet, F. Henrotte, L. Stainier, L.Noels and C. Geuzaine, An Energy-Based Variational Model of Ferromagnetic Hysteresis for Finite Element Computations, Journal of Computational and Applied Mathematics, in Press.

[9] G. Meunier, N. Charmoille, C. Gurin, P. Labie and Y. Marchal, Homogenization for Periodical Electromagnetic Structure: Which Formulation?, IEEE Transactions on Magnetics, vol. 42, no. 4, pp. 763-766, 2006.

[10] C. Miehe, Numerical Computational of Algorithmic (Consistent) Tangent Moduli in Large-Strain Computational Inelasticity, Computer Methods in Applied Mechanics and Engineering, vol. 134, pp. 223-240, 1996.

[11] I. Niyonzima, R. V. Sabariego, P. Dular and C. Geuzaine, Finite element computational homogenization of nonlinear multiscale materials in magnetostatics, IEEE Transactions on Magnetics, vol. 48, no. 2, pp. 587-590, 2012.

[12] D. Peric, E. A. Souza Neto, R. A. Feijoo, M. Partivo and A. J. Carneiro Molina, On micro-to-macro transitions for multiscale analysis of nonlinear heterogeneous materials: unified variational basis and finite element implementation, International Journal for Numerical Methods in Engineering, vol. 87, issue. 1-5, pp. 149-170, 2010.

[13] A. Visintin, Homogenization of Doubly-Nonlinear Equations, Rend. Lincei Mat. Appl., vol. 17, pp. 211-222, 2006.

[14] A. Visintin, Electromagnetic Processes in Doubly-Nonlinear Composites, Communications in Partial Differential Equations, vol. 33, pp. 804841, 2008. 